\title{
Consecuencias del síndrome de apnea obstructiva del sueño
}

\author{
MARÍA JOSÉ ELSO T. ${ }^{1,2}$, PABLO BROCKMANN V. ${ }^{3}$, DANIEL ZENTENO A., ${ }^{2,4}$ \\ 1. Neuróloga, Medicina del Sueño. Hospital Guillermo Grant Benavente, Concepción. \\ 2. Laboratorio de Sueño, Clínica Universitaria, Concepción. \\ 3. Pediatra Broncopulmonar, Medicina del Sueño.División de Pediatría, Escuela de Medicina, \\ Pontificia Universidad Católica de Chile. \\ 4. Pediatra Broncopulmonar. Departamento de Pediatría, Facultad de Medicina, Universidad de Concepción. \\ Hospital Guillermo Grant Benavente, Concepción.
}

\section{ABSTRACT \\ Consequences of obstructive sleep apnea syndrome in children}

The syndrome of obstructive sleep apnea (OSA) has emerged over the last decades as an important risk factor for heart and metabolic disease, and also, for neurocognitive dysfunction. The four major components of this condition are intermittent hypoxia, hypercapnia, intrathoracic pressure changes and sleep fragmentation. Several pathophysiological mechanisms are likely to be involved, such as increased oxidative stress, systemic inflammation and endothelial and autonomic dysfunction. Surgical treatment for OSA has been shown to be effective at normalizing endothelial function, reducing levels of inflammatory markers, and to improve the apnea-hypopnea index and sleep fragmentation, but this effect is smaller in obese children. The high prevalence of childhood obesity has changed the phenotypic expression of the disease, aggravating the problem and creating the need for effective interventions for this high-risk population. The aim of this study is to describe in detail the consequences of OSA on pediatric populations.

(Key words: Obstructive sleep apnea, consequences, children).

Rev Chil Pediatr 2013; 84 (2): 128-137

\section{RESUMEN}

El síndrome de apnea obstructiva del sueño (SAOS) ha emergido durante las últimas décadas como causa de morbilidad cardiaca, metabólica y disfunción neurocognitva. Los cuatro componentes mayores del SAOS son la hipoxia intermitente, hipercapnia, cambios de presión intratorácica y fragmentación de sueño. Los mecanismos fisiopatológicos incluyen el aumento del estrés oxidativo, inflamación sistémica, disfunción autonómica y endotelial. El tratamiento quirúrgico para el SAOS ha mostrado ser efectivo en normalizar la función endotelial, reduciendo los niveles de marcadores inflamatorios, el índice de apnea-hipopnea y mejorar la fragmen-

Recibido el 30 de mayo de 2012, devuelto para corregir el 07 de septiembre de 2012, segunda versión el 25 de octubre de 2012, aceptado para publicación el 11 de febrero de 2013.

Este trabajo cumple con los requisitos sobre consentimiento /asentimiento informado, comité de ética, financiamiento, estudios animales y sobre la ausencia de conflictos de intereses según corresponda.

Correspondencia a:

Daniel Zenteno A.

E-mail: danielzenteno@gmail.com 
tación de sueño, sin embargo este efecto seria menor en los niños obesos con SAOS. La alta prevalencia de obesidad infantil ha cambiado la expresión fenotípica de esta enfermedad, agravando el problema y establecido la necesidad de óptimas intervenciones para esta población de alto riesgo. El objetivo de este artículo es describir en profundidad las consecuencias del SAOS en población pediátrica.

(Palabras clave: Apnea obstructiva del sueño, consecuencias, niños).

Rev Chil Pediatr 2013; 84 (2): 128-137

\section{Consecuencias del síndrome de apnea obstructiva del sueño en niños}

El Síndrome de Apnea Obstructiva del Sueño (SAOS) es una condición altamente prevalente y se estima una frecuencia de 2 a $3 \%$ en niños ${ }^{1}$. Se caracteriza por la oclusión intermitente, parcial o completa de la vía aérea superior durante el sueño, produciendo alteración del intercambio gaseoso, frecuentes microdespertares y alteración de la arquitectura del sue$\tilde{\text { ño }}{ }^{2}$. El espectro de los trastornos respiratorios del sueño (TRS) incluye el SAOS, el síndrome de resistencia de la vía aérea superior y el ronquido primario. La principal causa de SAOS es la hipertrofia adenotonsilar; pero también la obesidad, las anomalías craneofaciales y las enfermedades neuromusculares son factores de riesgo y/o causas relacionadas con el desarrollo de $\mathrm{TRS}^{3}$. La obesidad contribuye de una forma muy importante en la fisiopatología del SAOS infantil, independientemente de la edad. La tabla 1 muestra la clasificación de las causas de los TRS.

La presentación clínica del SAOS es vaga, difiere de la presentación en adultos en varios aspectos por lo que requiere un alto índice de sospecha clínica. En niños sin comorbilidad asociada, la principal queja por parte de los padres es el ronquido habitual, definido por la presencia de ruido audible por más de tres noches por semana ${ }^{3}$. Dentro de los síntomas nocturnos, se describen aumento del esfuerzo respiratorio, apneas o pausas respiratorias, sueño intranquilo, posturas anormales al dormir para mantener una vía aérea permeable, enuresis y sudoración nocturna. Los síntomas diurnos incluyen sequedad bucal, obstrucción nasal asociada a respiración bucal, somnolencia diurna, hiperactividad y problemas neurocognitivos $^{5}$. La tabla 2 resume los principales signos y síntomas de SAOS en niños.

Se ha planteado la existencia de dos fenotipos, el primero de ellos, asociado a hipertrofia linfoadenoidea en ausencia de obesidad (tipo I) y el segundo, asociado a obesidad y con menor hiperplasia amigdalar (tipo II). Este último estaría sustituyendo progresivamente al patrón observado en la presentación fenotípica del SAOS en niños ${ }^{6}$.

La Polisomnografía (PSG) es considerada el estándar de oro como método de estudio que permite establecer la presencia y severidad de los TRS en niños, ya que registra las variables neurofisiológicas y cardio-respiratorias durante el sueño ${ }^{7}$. Un índice de apnea hipopnea $(\mathrm{IAH}) \geq 1,0$ permite definir la presencia de SAOS. La gravedad de SAOS se clasifica como leve con un IAH de 1 a 5, moderado con IAH entre 5 y 10 y SAOS grave con un IAH mayor a 10. La tabla 3 muestra los principales estudios de sueño disponibles y sus lugares más frecuentes de realización.

El Objetivo de este artículo es describir en

Tabla 1. Clasificación de causas de los trastornos respiratorios del sueño

\begin{tabular}{|lll|}
\hline TRS tipo I & TRS Tipo II & TRS Tipo III \\
Hipertrofia adenotonsilar sin obesidad & Obesidad, con leve a moderado & Enfermedades Neuromusculares \\
& hipertrofia adenotonsilar & S Down \\
& & Arnold Chiari \\
& & Paralisis cerebral \\
& & Síndromes craneofaciales: Pierre Robin, Apert, \\
\hline
\end{tabular}


Tabla 2. Signos y síntomas de SAOS en niños

\begin{tabular}{|ll|}
\hline Nocturnos & Diurnos \\
\hline - Ronquidos & - Respirador bucal \\
- Posturas anómalas al dormir & - Hiperactividad \\
- Sueño inquieto & - Disminución atencional \\
- Terrores nocturnos, sonambulismo & - Alteraciones conductuales \\
- Enuresis & - Somnolencia diurna* \\
- Sudoración nocturna & - Cefalea matinal \\
& - Sequedad bucal \\
& - Retraso pondoestatural \\
\hline
\end{tabular}

*Presente generalmente en adolescentes.

Tabla 3. Estudio de Sueño disponibles y sus lugares de realización

\begin{tabular}{|ll|}
\hline Estudio de Sueño & Lugar de realización \\
\hline a. Polisomnografía & Hospitalaria \\
\hline b. Poligrafía & $\begin{array}{l}\text { Domiciliaria preferentemente, hospitalaria } \\
\text { ocasionalmente }\end{array}$ \\
\hline c. Saturometría nocturna & $\begin{array}{l}\text { Domiciliaria preferentemente, hospitalaria } \\
\text { ocasionalmente }\end{array}$ \\
\hline
\end{tabular}

profundidad las consecuencias del SAOS en población Pediátrica; entregando así, argumentos que motiven al clínico a orientar su sospecha diagnóstica y su eventual manejo.

\section{Consecuencias cardiovasculares}

Estudios epidemiológicos han demostrado que el SAOS tanto en adultos como en niños está asociado a riesgo cardiovascular. El desarrollo de hipoxemia, de microdespertares recurrentes y de cambios de la presión intratorácica, desencadenaría una serie de respuestas, como el aumento del stress oxidativo, activación de la cascada inflamatoria sistémica, disfunción endotelial y activación simpática. Estos mecanismos serían los responsables de las consecuencias cardiovasculares, como la hipertensión arterial (HTA) y pulmonar, el remodelamiento cardiaco y la aterogénesis 9 .

Dado que no todos los pacientes desarrollan estas consecuencias mórbidas, se ha planteado un modelo de triple riesgo para explicar la variabilidad fenotípica. Esta sería el resultado final de la confluencia de la severidad de la enfermedad, de las condiciones ambientales y de la susceptibilidad genética individual ${ }^{3}$.

\section{Disfunción del sistema nervioso autonómico (SNA)}

Las alteraciones en el SNA conllevan un aumento de la descarga simpática, taquicardia, aumento de catecolaminas, disminución de la variabilidad de la frecuencia cardiaca (FC), aumento de la resistencia vascular sistémica y reducción del tono vagal $^{2}$. Debido a la dificultad de evaluar la función del SNA en niños, ya que se requieren estudios invasivos, se han diseñado pruebas no invasivas, como la velocidad de la onda de pulso, que mide el tiempo que tarda una pulsación en recorrer cierta distancia; la tonometría de pulso arterial, que corresponde a la respuesta del tono arterial a estímulos térmicos o respiratorios y el tiempo de tránsito de pulso que es el intervalo que tarda un pulso generado en el corazón hasta su llegada a territorios periféricos. La velocidad de la onda de pulso es considerado predictor del riesgo vascular ${ }^{10}$. Otra medida para evaluar la activación autonómica es la medición de catecolaminas séricas o urinarias. Snow et al demostraron un aumento de las catecolaminas urinarias en niños con SAOS y que se relacionaba directamente con la gravedad de la enfermedad ${ }^{11}$.

Muzumdar et al, evaluó las características autonómicas de 18 niños con SAOS mediante el estudio de la FC y del análisis de la variabilidad de esta, demostrando una reducción significativa de estos valores posterior al tratamiento y confirmando el aumento de la actividad simpática en el SAOS pediátrico. Sin embargo, 6 (33\%) de los 18 niños, persistieron con SAOS residual moderado a severo posterior a la adenotonsilectomía y solo logró ser erradicado completamente en 2 niños (11\%); sugiriendo que el manejo quirúrgico sería menos efectivo particularmente en asociación con obesidad ${ }^{12}$. 
Las alteraciones de la función autonómica parece ser la causa del incremento del tono arterial y de la consecuente aparición de HTA en niños con SAOS. Amin et al, realizó un monitoreo de $24 \mathrm{~h}$ de la PA en 140 niños, observando que en niños con SAOS leve y severo, existe una pérdida del fenómeno fisiológico de caída nocturna de la presión arterial, aumento de la variabilidad promedio de la PA en vigilia y sueño e incremento de la PA matutina, comparado con los controles, el cual se correlacionaba con el grado de severidad ${ }^{13}$.

Incluso los roncadores primarios presentan alteraciones de la función autonómica. Se han demostrado cambios significativos en la frecuencia cardíaca de niños con ronquido habitual comparado con sus pares no roncadores ${ }^{14}$.

\section{Disfunción endotelial}

Sumado a los cambios en el sistema nervioso autonómico, el SAOS induce activación de la cascada inflamatoria sistémica, produciendo daño estructural y funcional del endotelio ${ }^{15}$.

Gozal et al, estudiaron la función endotelial en 26 niños no obesos con SAOS y su respuesta al tratamiento. Demostraron un significativo compromiso de la función endotelial $\mathrm{y}$ aumento de los niveles de ligando soluble CD40 (sCD40L); marcador endotelial relacionado con la activación y disfunción endotelial (DE), en niños con SAOS comparado a los controles $(p<0,0001)$. Posterior a 6 meses de tratamiento con adenotonsilectomia, se observó una normalización de la función endotelial y reducción significativa de los niveles de SCD40L en la mayoría de los pacientes con SAOS. Sin embargo esta mejoría no fue observada en 6 niños con SAOS que presentaban antecedentes de historia familiar de enfermedad cardiovascular (ECV) prematura. Los autores plantean en primer lugar, que la inflamación sistémica es un importante contribuidor en la fisiopatología del SAOS y que la DE puede persistir luego del tratamiento, especialmente en niños con historia familiar de ECV. La habilidad de estos individuos de modular la respuesta inflamatoria sistémica podría estar alterada y predisponer a las consecuencias del SAOS $^{16}$.

En un estudio posterior se estudiaron los ni- veles de myeloid-related protein (MRP 8/14), los cuales estarían involucrados en la aterogénesis y cuyos niveles se correlacionarían con el grado de DE. Kim et al, observaron un aumento dosis-dependiente de los niveles de MRP 8/14 que se correlacionaban con la gravedad del SAOS, los cuales era más acentuado en presencia de obesidad. Por lo tanto la asociación entre obesidad y SAOS, amplificaría las respuestas inflamatorias sistémicas y la expresión de factores pro-aterogénicos, potenciando la magnitud del daño endotelial ${ }^{17}$.

Sin embargo, no todos los niños con SAOS desarrollan DE. Existen múltiples factores que podrían jugar un rol, principalmente la capacidad de reclutar mecanismos de reparación, como las células progenitoras endoteliales (CPE). Las CPE tienen el potencial de revertir el daño vascular y su cantidad circulante podría ser un importante factor en determinar la magnitud de la $\mathrm{DE}^{18}$. Un estudio reciente evaluó 80 niños no hipertensos pre-púberes con SAOS confirmado por PSG y 20 controles. Fueron sometidos a test de hiperemia modificada, medición de niveles de CPE y de células estromales derivadas del factor-1 (SDF-1). Demostraron que los niveles de CPE fueron significativamente más bajos en SAOS asociado a DE comparado con los controles, pero significativamente más altos que aquellos niños con SAOS sin DE. Al contrario, los niveles de SDF-1 eran mayores en SAHOS sin DE y similar a los controles ${ }^{18}$.

\section{Inflamación sistémica y aterogénesis}

Existe evidencia de la fuerte asociación entre SAOS y la aterogénesis, en la que participarían fenómenos inflamatorios sistémicos. El modelo propuesto plantea que el SAOS promovería la aterogénesis, en primer lugar, mediante la formación y liberación de citoquinas inflamatorias, como interleucina-6 (IL-6), interleucina-1 (IL-1), factor necrosis tumoral alfa (TNF-alfa), leptinas y adipoquinas desde el tejido adiposo. Además aumentaría el estrés oxidativo vía formación y liberación de peróxido de hidrógeno, especies nitrógeno reactivas y aumento de reactantes de fase aguda desde el hígado. Por otro lado, disminuiría la expresión del óxido nítrico sintetasa endotelial, con au- 
mento de moléculas de adhesión, apoptosis de células endoteliales y activación de plaquetas. Se plantea que el monocito es la célula principalmente afectada por las condiciones de hipoxia-reoxigenación, resultando en un aumento de macrófagos activos, induciendo cascada de citoquinas y factores de crecimiento. Estos promoverían la proliferación de células musculares lisas en la pared vascular, la migración de macrófagos a través del endotelio dañado y su transformación en células espumosas, considerada la célula prototipo de la lesión ateromatosa inicial ${ }^{19}$.

Por otra parte, se plantea que el estrés oxidativo secundario a la hipoxia intermitente conduciría a la activación de linfocitos $\mathrm{T}^{20}$. Ambas células T CD4 y CD8 están activadas en pacientes con SAOS y muestran un aumento en la expresión de interleucina-4 (IL-4) y reducción de la interleucina-10 (IL-10), considerada esta última, una molécula antiinflamatoria ${ }^{21}$.

Proteínas pro-inflamatorias como proteína C-reactiva (PCR) e IL-6 son considerados marcadores de ECV. Gozal et al, analizaron las respuestas inflamatorias sistémicas en niños no obesos con SAOS, observando un significativo aumento de los niveles plasmáticos de IL-6 $(\mathrm{p}<0,01)$ y disminución de IL-10 $(\mathrm{p}<0,001)$ comparado a los controles, normalizándose posterior a la adenotonsilectomia ${ }^{22}$. Así también en otro estudio, demostraron una disminución significativa de los niveles elevados de PCR en niños con SAOS luego de 14 semanas posterior al tratamiento quirúrgico $(\mathrm{p}<, 05)^{23}$.

El SAOS induce un estado de hipercoagulabilidad. Estudios han confirmado la elevación del fibrinógeno matutino en SAOS pediátrico $^{24}$. La p-selectina es una molécula de adhesión plasmática, considerado un marcador de activación plaquetaria. O'Brien et al, demostró que los niveles de p-selectina en niños con SAOS estaban significativamente aumentados en comparación a los controles, aún en sus formas leves $(p<0,001)^{25}$. Sugiriendo que ya la presencia de SAOS leve puede afectar la expresión de marcadores de superficie en las plaquetas circulantes y aumentar el riesgo de morbilidad cardiovascular en niños genéticamente susceptibles.

\section{Consecuencias metabólicas}

El aumento de la prevalencia de SAOS parece estar relacionado con el aumento de la prevalencia de la obesidad. Los mecanismos fisiopatológicos incluirían factores anatómicos, neuromusculares, alteraciones en los mecanismos de la pared torácica y del control ventilatorio $^{4}$. El riesgo de padecer SAOS en niños obesos es de 4 a 5 veces mayor y el riesgo aumenta en un $12 \%$ por cada aumento en un $1 \mathrm{~kg} / \mathrm{m} 2$ en el $\mathrm{IMC}^{26}$.

La asociación entre SAOS y obesidad parece amplificar las alteraciones metabólicas y la interrelación entre ambas sería bidireccional, amplificando las respuestas inflamatorias y facilitando las consecuencias adversas de $\operatorname{ambas}^{27}$

El retraso pondoestatural es otra complicación asociada al SAOS. Sin embargo, se reporta una incidencia menor al 5\% de niños con SAOS e incluso aquellos con obesidad presentan ganancia de peso posterior al tratamiento $^{28}$. Los mecanismos propuestos son la disminución de los niveles de factor de crecimiento insulínico tipo 1 (IGF-1), de la proteína transportadora 3 del factor de crecimiento insulínico (IGFBP-3) y probablemente de la liberación de hormona de crecimiento $^{29}$. Además existe aumento del riesgo de esteatosis hepática no alcohólica en niños con SAOS y obesidad principalmente asociados a síndrome metabólico (SM), y con mejoría de los niveles de transaminasas luego del tratamiento ${ }^{30}$.

Así también la presencia de SAOS puede promover o agravar la obesidad. El SAOS se asocia a somnolencia diurna excesiva (SDE), promoviendo la inactividad física, por tanto la ganancia de peso y obesidad. Los niños obesos con SAOS tienen 4,2 menos probabilidad de realizar deportes y muestran aumentos significativos de los niveles plasmáticos de grelina, hormona asociada al aumento de apetito, comparado con niños no obesos con SAOS y obesos $\sin$ SAOS $(\mathrm{p}<0,001)^{31}$. Por otro lado, los niños obesos presentan un alto riesgo de desarrollar TRS, desde ronquido habitual hasta apneas obstructivas del sueño y apneas centrales $^{32}$. Sin embargo, Kohler et al, plantean que no existe evidencia suficiente que demuestre 
una relación directa entre obesidad y SAOS, ya que otros factores como edad, etnia y morfología craneofacial influirían en su génesis ${ }^{33}$.

Estudios han confirmado que la obesidad promueve la probabilidad de SDE en niños con SAOS. Gozal et al, comparó un grupo de 55 niños pre-púberes obesos y 50 niños no obesos con SAOS evaluados con PSG y test de latencias múltiples de sueño (TLMS). Observó que las latencia promedio de sueño estaban significativamente reducidas en obesos comparados con los no obesos con SAOS (TLMS 12,9+ $\left.0,8 \min v_{s} 17,9+0,9 \min , \mathrm{p}<, 00005\right)$. Así también, 21 niños obesos presentaba un TLMS alterado $(<12 \mathrm{~min})$, indicativo de somnolencia patológica, en comparación a solo 5 niños no obesos, demostrándose una asociación significativa entre las latencias promedio de sueño y el IMC $(p=0,0001)^{34}$.

Los niveles de TNF- $\alpha$ podrían considerarse un marcador biológico de SDE. Un estudio reciente reportó que las concentraciones plasmáticas matinales de TNF- $\alpha$ estaban significativamente más elevadas en niños con SAOS en comparación a los controles y además se correlacionaban principalmente con el IAH, la fragmentación de sueño y el IMC. El aumento de niveles de TNF- $\alpha$ se correlacionaba con el acortamiento patológico de las latencias promedio de sueño y la mejoría de ambos parámetros posterior a la adenotonsilectomía ${ }^{35}$.

Khalyfa et al, demostraron que la presencia o ausencia de polimorfismo específicos del gen de TNF- $\alpha$ se asocia a la variabilidad de los niveles de TNF- $\alpha$ y en consecuencia a la expresión fenotípica de la SDE en $\mathrm{SAOS}^{36}$.

La adenotonsilectomia también ha demostrado mejorar el IAH y la fragmentación de sueño, principalmente en niños no obesos. Además de la reducción significativa en el perfil lipídico, en los niveles de PCR y de apolipoproteína-B tanto en niños obesos como no obesos. En niños obesos, el tratamiento quirúrgico se asoció a una mejoría significativa de la resistencia insulina (RI), independiente de los cambios de IMC, por lo tanto el SAOS en presencia de obesidad, podría jugar un rol en el control glicémico ${ }^{37}$. Sin embargo, Tauman et al, no encontraron asociación entre la RI y gravedad, planteando que la dislipidemia y la RI estaría determinado más por el IMC que por la severidad del SAOS ${ }^{38}$. Cabe destacar que la adenotonsilectomía ha demostrado tener una alta tasa de falla en pacientes obesos, en los cuales el único tratamiento que impresionaría efectivo sería la disminución del IMC y de la grasa corporal. Un estudio multicéntrico analizó el resultado del tratamiento del SAOS en 578 niños, de los cuales el 50\% de ellos eran obesos. Demostró que sólo un 27,2\% presentaban resolución completa posterior a la adenotonsilectomía, existiendo una alta proporción con SAOS residual, especialmente en niños mayores ( $>7$ años) y en obesos ${ }^{39}$.

Nahra et al, evaluaron a 25 niños con síndrome metabólico y SAOS diagnosticados con PSG. Observaron que los niveles plasmáticos de leptina y norepinefrina eran significativamente mayores que los controles y que disminuían luego de 3 meses de tratamiento con ventilación mecánica no invasiva, mediante presión positiva continua en la vía aérea ${ }^{40}$.

Dayyat et al investigaron la contribución de la obesidad y la hipertrofia adenotonsilar en la génesis del SAOS. Estudiaron a 206 niños no obesos y 206 niños obesos con SAOS, evaluados con PSG, estimación del tamaño adenotonsilar y score de Mallampati. Demostraron que la magnitud de la hipertrofia adenotonsilar para la misma gravedad de SAOS, es menor en niños obesos comparado con los no obesos, sin embargo los scores de Mallampati estaban considerablemente aumentados en los niños obesos. Estos hallazgos confirman que la obesidad facilitarían la ocurrencia de SAOS en presencia de hipertrofia adenotonsilar, debido a que los depósitos grasos subcutáneos en la región cervical anterior producirían estrechamiento de la vía aérea superior, facilitando su colapso $^{41}$. Otros estudios han confirmado que tanto factores anatómicos como funcionales están involucrados en la fisiopatología, dado que aproximadamente un $50 \%$ de los niños obesos sometidos a adenotonsilectomia persisten con SAOS residual en comparación con un 10 a $20 \%$ de los niños no obesos ${ }^{4}$.

El advenimiento de la sociedad moderna y de la tecnología ha producido alteraciones de los patrones de sueños tanto en adultos como en niños. La progresiva disminución del tiem- 
po total de sueño se ha visto acompañado por un aumento sucesivo de la obesidad. La privación crónica de sueño se traduciría en alteraciones de los neuropéptidos que regulan el apetito, produciendo aumento de los niveles de glerina y disminución de los niveles de leptina y de la actividad central de orexina, favoreciendo el aumento de la ingesta alimentaria ${ }^{26}$. Un estudio reciente demostró que al prolongar en una hora por noche el sueño nocturno, reduce en un $9 \%$ el riesgo de un aumento del $\mathrm{IMC}^{42}$.

\section{Consecuencias Neurocognitivas}

Uno de las más importantes consecuencias del SAOS son las alteraciones neurocognitivas en niños, que incluyen problemas de aprendizaje, con disminución del rendimiento escolar, déficit en la atención, memoria y funciones ejecutivas; alteraciones conductuales como hiperactividad, irritabilidad y agresividad y alteraciones del ánimo ${ }^{10,43}$.

Se ha propuesto que las alteraciones neuroconductuales y cognitivas sería secundarias a la disfunción en los procesos dependientes del cortex prefrontal inducida por los fenómenos de hipoxemia ${ }^{44}$. La hipoxemia se relacionaría con el déficit en la función ejecutiva y la SDE con el déficit atencional ${ }^{45}$. Actualmente se postula que la principal alteración seria el proceso de fijación deficiente de la memoria ${ }^{46}$.

Sin embargo no todos los niños presentan alteraciones cognitivas, planteándose otros factores asociados, como la obesidad, que podría aumentar la vulnerabilidad cognitiva ${ }^{47}$. Gozal et al, estudiaron a 87 niños entre 5 y 8 años con SAOS mediante baterías de evaluación cognitiva y medición de la función endotelial. En 48 niños con disfunción neurocognitiva, 40 de ellos además presentaban DE $(83,3 \%)$ Así también en 50 niños con $\mathrm{DE}, 40$ presentaban compromiso cognitivo $(80 \%)$, demostrando coexistencia entre ambas alteraciones, por lo cual plantean que estas consecuencias mórbidas compartirían mecanismos patogénicos ${ }^{48}$.

La SDE es uno de los síntomas principales de alteración del sueño nocturno. Es importante recalcar que la presentación clínica de la SDE difiere entre adultos y niños, ya que estos últimos se manifiestan principalmente como hiperactividad y que este estado de hipervigilia sería más bien el reflejo de una SDE enmascarada ${ }^{47}$. Solamente un $7 \%$ de los padres reportan síntomas compatibles con $\mathrm{SDE}$ en niños con sospecha de TRS ${ }^{49}$. Por otro lado, la medición objetiva de la SDE, reporta que la SDE alcanza entre un 13 a $20 \%$ y parece ser más frecuente en niños obesos ${ }^{50}$.

La comorbilidad entre el síndrome de déficit atencional con hiperactividad (SDAH) y TRS es alta $(23 \%)^{51}$. Así también el ronquido habitual no sería un proceso benigno, ya que se ha reportado mayor frecuencia de SDE, hiperactividad, problemas de aprendizaje y sueño inquieto que en niños sanos ${ }^{52}$.

Un meta análisis analizó 61 estudios confirmando que los TRS en niños se asocian a déficit en la regulación emocional y conductual, en el rendimiento escolar, en la atención sostenida y alerta. Una menor evidencia apoyaría su asociación con alteraciones del ánimo, habilidades del lenguaje expresivo, percepción visual y memoria de trabajo ${ }^{53}$. Un estudio prospectivo posterior con 20 niños en cada grupo de caso y control entre 3 y 12 años, mostró una diferencia estadísticamente significativa en atención, memoria y estructuración espacial ${ }^{54}$. Así también existe evidencia que documenta significativa reducción en la medición del coeficiente intelectual en niños con SAOS comparado a los controles.

Se ha demostrado la mejoría de las alteraciones cognitivas y conductuales posterior al tratamiento de SAOS, lo que sugiere que al menos algunos de los déficit serían reversibles, especialmente si el manejo es temprano y efectivo ${ }^{47}$. Un estudio evaluó a 297 niños que correspondían al percentil 10 más bajo de rendimiento escolar, realizando tamizaje para TRS y evaluación de la gasometría nocturna, reportando alteración en 54 niños $(18,1 \%)$. De estos, en 24 niños se realizó tratamiento quirúrgico y en 30 de ellos no se realizó intervención. El mayor aporte de este estudio es que evaluaron a este grupo de niños 1 año después y observaron, que en los 24 niños sometidos a cirugía y corregido su cuadro, se observó un aumento estadísticamente significativo en el rendimiento escolar $(\mathrm{p}<, 001)$, a diferen- 
cia de los niños sin intervención, en los que no se modificó su rendimiento ${ }^{55}$. Un estudio más reciente, demuestra que el puntaje en la evaluación neurocognitiva es menor en niños con TRS que en los controles y que estos mejoran significativamente posterior a la cirugía, asociado a una mejoría en la arquitectura del sueño, aumento del sueño de ondas delta y mejoría en $\mathrm{IAH}^{56}$.

Por lo tanto sería fundamental la pesquisa de pacientes con sospecha de SAOS, en etapas tan precoces, como el primer año de vida. Bonuck et al, estudiaron la presencia de síntomas de TRS entre los 6 y 69 meses de edad, en más de 11.000 niños, demostrando que los TRS serían predictores del desarrollo de alteraciones conductuales, como hiperactividad y dificultades emocionales, en un 40 a $60 \%$ entre 4 y 7 años de edad ${ }^{57}$.

\section{Conclusiones}

Actualmente existe suficiente evidencia para afirmar que el SAOS es una enfermedad inflamatoria sistémica y posee evidentes consecuencias cardiovasculares, metabólicas y neurocognitivas en niños; principalmente secundarias al stress oxidativo y las respuestas inflamatorias.

Aunque el tratamiento quirúrgico ha mostrado ser efectivo en disminuir o normalizar la función endotelial, reducir los niveles de marcadores inflamatorios, mejorar el perfil lipídico, el IAH y la fragmentación de sueño, parece no ser tan efectivo en niños obesos con SAOS.

A medida que observemos un aumento mundial de la prevalencia en la obesidad infantil, seremos testigos de un aumento de casos de SAOS infantil y de la interacción bidireccional entre ambas. La frecuencia y severidad de las consecuencias cardiovasculares será amplificada por la asociación con obesidad, probablemente asemejándose a las complicaciones observadas en adultos; sin embargo, desconocemos cuáles serán las implicancias a futuro de la coexistencia de ambas patologías.

Durante la infancia es el periodo en el cual el cerebro está en su máximo desarrollo por lo cual cualquier injuria en sus procesamientos normales impacta en forma negativa su neurodesarrollo. Así también, es importante enfatizar la necesidad de mantener una adecuada cantidad y calidad de sueño en niños, medida que podría jugar un rol importante en mitigar los efectos adversos principalmente en el aspecto neurocognitivo en esta población.

\section{Referencias}

1.- Lumeng J, Chervin R: Epidemiology of pediatric obstructive sleep apnea. Proc Am Thorac Soc 2008; 5: 242-52.

2.- Bhattacharjee R, Kheirandish-Gozal L, Pillar G, Gozal $D$ : Cardiovascular complications of obstructive sleep apnea syndrome: evidence from chidren. Prog Cardiovasc Dis 2009; 51: 416-33.

3.- Gozal D, Kheirandish L: Oxidant stress and inflammation in the snoring child: confluent pathways to upper airway pathogenesis and end-organ morbidity. Sleep Med Rev 2006; 10:83-96.

4.- Arens R, Muzumdar H: Childhood obesity and obstructive sleep apnea syndrome. J Appl Physiol 2010; 108: 436-44.

5.- Prades-Morera E, Esteller Moré E: Clinica de los trastornos respiratorios del sueño en niños. Acta Otorrinolaringol Esp 2010; 61: 22-5.

6.- Gozal D, Kheirandish-Gozal L: New approaches to the diagnosis of sleep-disordered breathing in children. Sleep Med 2010; 11:708-13

7.- Katz E, D’Ambrosio C: Pediatric Obstructive sleep apnea síndrome. Clin Chest Med 2010; 31: 221-34.

8.- American Academy of Pediatrics. Clinical Practice Guideline: Diagnosis and management of childhood obstructive sleep apnea syndrome. Pediatrics 2002; 109: 704-12.

9.- Gozal D: Sleep, sleep disorders and inflamation in children. Sleep Med 2009; 10: S12-6.

10.- Alonso-Álvarez M, Canet T, Cubell-Alarco C, et al: Documento de Consenso de Síndrome de Apneas-hiponeas durante el sueño en niños. Arch Bronconeumol 2011; 47: 2-18.

11.- Snow A, Khalyfa A, Serpero L, et al: Cathecholamine in alterations in pediatric obstructive sleep apnea: effect of obesity. Pediatr Pulmonol 2009; 44: 559-67.

12.- Muzumdar HV, Sin S, Nikova M, Gates G, Kim D, Arens $R$ : Changes in heart rate variability after adenotonsillectomy in children with obstructive sleep apnea. Chest 2011; 139: 1050-9.

13.- Amin R, Somers V, McConnell K, et al: Activity- 
adjusted 24-hour ambulatory blood pressure and cardiac remodeling in children with sleep disordered breathing. Hypertension 2008; 51: 84-91.

14.- Brockmann P, Urschitz M, Noehren A, Sokollik C, Schlaud M, Poets $C$ : Risk factors and consequences of excessive autonomic activation during sleep in children. Sleep Breath 2011; 15: 409-16.

15.- Kheirandish-Gozal L, Bhattacharjee R, Gozal P: Autonomic alterations and endothelial dysfunction in pediatric obstructive sleep apnea. Sleep med 2010; 11: 714-20.

16.- Gozal D, Kheirandish-Gozal L, Serpero L, Sans Capdevila O, Dayyat E: Obstructive sleep apnea and endothelial function in school-aged nonobese children: effect of adenotonsillectomy. Circulation 2007; 116 : 2307-14.

17.- Kim J, Bhattacharjee R, Snow A, Kheirandish-Gozal L, Gozal D: Myeloid related protein 8/14 levels in children with obstructive apnea. Eur Respir J 2010; 35: 843-50.

18.- Kheirandish-Gozal L, Bhattacharjee R, Kim J, Clair $H$, Gozal D: Endothelial dysfunction in children with obstructive sleep apnea. Am J Respir Crit Care Med 2010; 182: 92-7.

19.- Gozal D, Kheirandish-Gozal L: Cardiovascular morbidity in obstructive sleep apnea: oxidative stress, inflammation, and much more. Am J Respir Crit Care Med 2008; 177: 369-75.

20.- De la Peña M, Barceló A, Barbé F, Piérola J, Pons J, et al: Endothelial function and circulating endothelial progenitor cells in patients with sleep apnea syndrome. Respiration 2008; 76:28-32.

21.- Dyugovskaya L, Lavie P, Hirsh M, Lavie L: Activated CD8+ T-lymphocytes in obstructive sleep apnea. Eur Respir J 2005; 25: 820-82.

22.- Gozal D, Serpero L, Capdevila OS, Kheirandish-Gozal $L$ : Systemic inflammation in non-obese children with obstructive sleep apnea. Sleep Med 2008; 9: 254-9.

23.- Kheirandish-Gozal L, Capdevila OS, Tauman R, Gozal $D$ : Plasma C-reactive protein in nonobese children with obstructive sleep apnea before and after adenotonsillectomy. J Clin Sleep Med 2006; 2: 301-4.

24.- Kaditis A, Alexopoulos E, Kalampouka E, et al: Morning levels of fibrinogen in children with sleep disordered breathing. Eur Res J 2004; 24: 790-7.

25.- O'Brien L, Serpero L, Tauman R, Gozal D: Plasma adhesion molecules in children with sleep-disordered breathing, Chest 2006; 129: 947-53.

26.- Redline S, Tishler PV, Schluchter M, et al: Risk factors for sleep-disordered breathing in children: associations with obesity, race, and respiratory problems. Am J Res- pir Crit Care Med 1999; 159: 1527-32.

27.- Gozal D, Kheirandish-Gozal L: The obesity epidemic and disordered sleep during childhood and adolescence. Adolesc Med 2010; 2: 480-90.

28.- Roemmich J, Barkley J, D’ Andrea L, et al: Increases in overweight after adenotonsillectomy in overweight children with obstructive sleep-disordered breathing are associated with motor activity and hyperactivity .Pediatrics 2006; 117: 200-8.

29.- Nieminen P, Löppönen T, Tolonen U, Laining P, Knip $M$ : Crecimiento y marcadores bioquímicos del mismo en niños con ronquidos y apnea obstructiva del sueño. Pediatrics 2002 (ed esp); 53: 225-33.

30.- Kheirandish-Gozal L, Capdevilla O, Kheirandish E, Gozal D: Elevated serum aminotransferase levels in children at risk for obstructive sleep apnea. Chest 2008; 133: 92-9.

31.- Spruyt K, San Capdevila O, Honaker S, Bennett J, Gozal D: Obesity, snoring and physical activity in schoolaged children. Sleep 2008; 31: A76.

32.- Verhulst S, Van Gaal, De Backer W, Desager K: The prevalence, anatomical correlates and treatment of sleep-disordered breathing in obese children and adolescents. Sleep Med Reviews 2008; 12: 339-46.

33.- Kohler M, Heuvel $C$ : Is there a clear link between overweight/obesity and sleep disordered breathing in children?. Sleep Med Reviews 2008; 12: 347-61.

34.- Gozal D, Kheirandish-Gozal L: Obesity and excessive daytime sleepiness in prepubertal children with obstructive sleep apnea. Pediatrics 2009; 123: 13-8.

35.- Gozal D, Serpero L, Kheirandish-Gozal L, et al: Sleep measures and morning plasma TNF- $\alpha$ levels in children with sleep-disorderes breathing Sleep 2010; 33: 319-25.

36.- Khalyfa A, Serpero L, Kheirandish-Gozal L, Capdevilla $O$, Gozal D: TNF- $\alpha$ gene polymorphisms and excessive daytime sleepiness in pediatric obstructive sleep apnea. J Pediatr 2011; 158: 77-82.

37.- Gozal D, Capdevila OS, Kheirandish-Gozal L: Metabolic alterations and systemic inflammation in obstructive sleep apnea among nonobese and obese prepubertal children. Am J Respir Crit Care Med 2008; 177: 11429.

38.- Tauman R, O'Brien L, Ivanenko A, Gozal D: Obesity rather than severity of sleep-disordered breathing as the mayor determinant of insulin resistance and altered lipidemia in snoring children. Pediatrics 2005; 116: 6673.

39.- Bhattacharjee R, Kheirandish-Gozal L, Spruyt K, et al: Adenotonsillectomy outcomes in treatment of obstructive sleep apnea in children. Am J Respir Crit Care Med 
2010; 182: 676-83.

40.- Nakra N, Bhargava S, Dzuira J, Caprio S, Bazzy-Asaad $A$ : Sleep-Disordered breathing in children with metabolic syndrome: the role of leptin and sympathetic nervous system activity and the effect of continuos positive airway pressure. Pediatrics 2008; 122: 634-40.

41.- Dayyat E, Kheirandish-Gozal L, Sans Capdevilla O, Maarafeya M, Gozal D: Obstructive sleep apnea in children: relative contributions of body mass index and adenotonsillar hypertrophy. Chest 2009; 136: 137-44.

42.- Chen X, Beydoun M, Wang Y: Is sleep duration associated with childhood obesity? A systematic review and meta-analysis. Obesity 2008; 16: 265-74.

43.- Owens J: Neurocognitive and behavioral impact of sleep disordered breathing in children. Pediatric Pulmonology 2009; 44: 417-22.

44.- Beebe D, Gozal D: Obstructive sleep apnea and the prefrontal cortex: towards a comprehensive model linking nocturnal upper airway obstruction to daytime cognitive and behavioural deficits. J Sleep Res 2002; 11: 1-16.

45.- Bedard M, Montplaisir J, Richer F, Rouleau I, Malo $J$ : Obstructive sleep apnea syndrome: pathogenesis of neuropsychological deficits. J Clin Exp Neuropsychol 1991; 13: 950-64.

46.- Spruyt K, Capdevilla O, Kheirandish-Gozal L, et al: Inefficient or insufficient encoding as potencial primary deficit in neurodevelopmental performance among children with OSA. Dev Neuropsychol 2009; 34: 601-14.

47.- Gozal D: Obstructive sleep apnea in children: implications for the developing central nervous system. Semin Pediatr Neurol 2008; 15: 100-6.

48.- Gozal D, Kheirandish-Gozal L, Bhattacharjee R, Spruyt
$K$ : Neurocognitive and endothelial dysfunction in children with obstuctive sleep apnea. Pediatrics 2010; 126 : 1161-7.

49.- Carrol J, McColley S, Marcus C, Curtis S, Loughlin G: Inability of clinical history to distinguish primary snoring from obstructive sleep apnea syndrome in children. Chest 1995; 108: 610-8.

50.- Gozal D, Wang M, Pope D: Objective sleepiness measures in pediatric obstructive sleep apnea. Pediatrics 2001; 108: 693-7.

51.- Owens J: Sleep disorders and attention-deficit/hyperactivity disorder. Curr Psychiatry Rep 2008; 10: 439-44.

52.- O'Brien L, Holbrook C, Mervis C, et al: Sleep and neurobehavioral charaxteristics of 5-to-7-year- old children with parentally reported symtoms of attention-deficit/ hyperactivity disorder. Pediatrics 2003; 111: 554-63.

53.- Beebe D: Neurobehavioral morbidity associated with disordered breathing during sleep in children: a comprehensive review. Sleep 2006; 29: 1115-34.

54.- Esteller E, Barceló M, Segarra F, et al: Alteraciones neurocognitivas y conductuales en los trastornos respiratorios del sueño infantil. Acta Otorrinolaringol Esp 2009; 60: 325-31.

55.- Gozal D: Sleep-disordered breathing and school performance in children. Pediatrics 1998; 102: 616-20.

56.- Montgomery-Downs H, Crabtree V, Gozal D: Cognition, sleep, and respiration in at- risk children treated for obstructive sleep apnoea. Eur Respir J 2005; 25: 336-42.

57.- Bonuck K, Freeman K, Chervin R, Xu L: SleepDisordered breathing in a population-based cohort: behavioural outcomes at 4 and 7 years. Pediatrics 2012; 129: 857-65. 